\title{
Serum Uric Acid Is Associated with Poor Outcome in Black Africans in the Acute Phase of Stroke
}

\author{
Yacouba N. Mapoure, ${ }^{1,2}$ Chia Mark Ayeah, ${ }^{2}$ M. S. Doualla, ${ }^{2,3}$ H. Ba, ${ }^{3}$ \\ Hugo B. Mbatchou Ngahane, ${ }^{1,2}$ Salomon Mbahe, ${ }^{2}$ and Henry N. Luma ${ }^{2,3}$ \\ ${ }^{1}$ Department of Clinical Sciences, University of Douala, Douala, Cameroon \\ ${ }^{2}$ Department of Internal Medicine, Douala General Hospital, Douala, Cameroon \\ ${ }^{3}$ Department of Internal Medicine, University of Yaoundé I, Douala, Cameroon \\ Correspondence should be addressed to Yacouba N. Mapoure; mapoureyacouba@gmail.com
}

Received 17 June 2017; Revised 16 July 2017; Accepted 24 July 2017; Published 10 September 2017

Academic Editor: David S. Liebeskind

Copyright (C) 2017 Yacouba N. Mapoure et al. This is an open access article distributed under the Creative Commons Attribution License, which permits unrestricted use, distribution, and reproduction in any medium, provided the original work is properly cited.

\begin{abstract}
Background. Prognostic significance of serum uric acid (SUA) in acute stroke still remains controversial. Objectives. To determine the prevalence of hyperuricemia and its association with outcome of stroke patients in the Douala General Hospital (DGH). Methods. This was a hospital based prospective cohort study which included acute stroke patients with baseline SUA levels and 3month poststroke follow-up data. Associations between high SUA levels and stroke outcomes were analyzed using multiple logistic regression and survival analysis (Cox regression and Kaplan-Meier). Results. A total of 701 acute stroke patients were included and the prevalence of hyperuricemia was $46.6 \%$ with a mean SUA level of $68.625 \pm 24 \mathrm{mg} / \mathrm{l}$. Elevated SUA after stroke was associated with death $(\mathrm{OR}=2.067 ; 95 \%$ CI: 1.449-2.950; $p<0.001)$ but did not predict this issue. However, an independent association between increasing SUA concentration and mortality was noted in a Cox proportional hazards regression model (adjusted HR = 1.740; 95\% CI: $1.305-2.320 ; p<0.001)$. Furthermore, hyperuricemia was an independent predictor of poor functional outcome within 3 months after stroke $(\mathrm{OR}=2.482 ; 95 \% \mathrm{CI}: 1.399-4.404 ; p=0.002)$. Conclusion. The prevalence of hyperuricemia in black African stroke patients is quite high and still remains a predictor of poor outcome.
\end{abstract}

\section{Introduction}

Stroke is the second most common cause of death and major cause of disability worldwide [1]. The burden of stroke is increasing in many low- and middle-income countries (LMIC) [2], and due to the high fatality rates and overwhelming resources incurred by health systems, stroke and many noncommunicable diseases (NCDs) are now targeted public health priorities in these regions $[3,4]$. SUA is one of the most important antioxidants abundant in the plasma, contributing to approximately two-thirds of free radical scavenging in plasma $[5,6]$. SUA has been concerned as a free radical scavenger protecting nerves from oxidative injury $[7,8]$. A study explored the relationship between SUA levels and 1-year outcomes, and vascular events of acute ischemic stroke patients showed that decreased SUA levels were related to poor outcomes and had a higher risk of all-cause death [9]. Meanwhile, Amaro et al. suggested that increased SUA is associated with better outcomes in patients with stroke treated with reperfusion therapies and supported the assessment of the potential neuroprotective role of the exogenous administration of UA in patients with stroke [10]. These findings suggested that SUA may be neuroprotective in acute ischemic stroke patients. Conversely, some studies have explored the relationship between SUA and stroke prognosis but have had contradicting results. Previous experimental studies in recent years have reported a relationship between SUA and several cardiovascular conditions including acute myocardial infarction (AMI), congestive heart failure (CHF), and both ischemic and hemorrhagic stroke [11-14]. These studies suggested that increasing SUA was associated with a higher risk of mortality. Despite previous and current studies, the neuroprotective role of SUA in stroke remains controversial. In this present study, our objectives were to 
determine the prevalence of hyperuricemia and to examine the association of SUA levels with CVRFs and outcomes of stroke patients in the acute phase.

\section{Methods and Patients}

2.1. Patients and Study Design. We carried out a hospital based prospective cohort study in a tertiary care hospital in Douala, Cameroon. We included consenting patients admitted for acute stroke (both ischemic and hemorrhagic) in the Neurology Unit of the Department of Internal Medicine and the Intensive Care Unit of the Douala General Hospital (DGH) from January 2010 to January 2016. This study was approved by the Institutional Ethics Committee of Research on Human Health of the University of Douala and the study hospital DGH. Patients who were admitted for confirmed acute stroke within 7 days of onset of symptoms were included in our study. Patients with cerebral venous thrombosis and subarachnoid hemorrhage were excluded.

\subsection{Data Collection and Patient Management. Demographic} data, including age and sex, and relevant medical history such as hypertension (HTN), diabetes mellitus (DM), smoking history, alcohol abuse, use of diuretics, history of diseases like chronic kidney disease (CKD), and gout and other cardiovascular events such as atrial fibrillation, congestive heart failure (CHF), coronary artery disease (CAD), and ischemic heart disease (IHD) were recorded. Baseline vital and anthropometric parameters such as blood pressure, pulse, respiratory rate, oxygen saturation, weight, height, and abdominal circumference values were recorded using standard operating procedures. Hypertension, diabetes mellitus, dyslipidemia, alcohol consumption, and obesity were defined as in the previous study published in this journal by Mapoure et al. in 2014 [15]. Metabolic syndrome was defined as per the NCEP ATP III guidelines [16]. Patients with severe conditions like a Glasgow Coma Scale $<8 / 15$ or septic shock were directly admitted in the ICU while other cases were hospitalized in the NU.

Blood samples were collected from all patients during the first 24 hours of admission to check SUA levels, fasting blood sugar, complete metabolic panel (urea, creatinine, uric acid, and electrolytes), and lipid profile using the Cobas 311 autoanalyzers. A full blood count with platelet counts, prothrombin time, kaolin cephalin clotting time, C-reactive protein (CRP), erythrocyte sedimentation rate (ESR), and HIV serology was done. Other tests were prescribed if required by the patient's conditions: chest X-ray, urine culture, hemoculture, and thick blood film to check for Plasmodium falciparum. Neurological assessment was done by a neurologist or intensive care specialist. Interpretation of CT scans was done by both a radiologist and a neurologist and its findings were recorded. The type of stroke and ischemic stroke subtypes was also recorded. Electrocardiography was systematically done for patients with ischemic stroke and for hypertensive patients with hemorrhagic strokes. For patients with ischemic stroke, transthoracic and supra-aortic Doppler ultrasound was done, except for those with severe conditions. On admission and at 3 months after stroke, the Glasgow
Coma Scale (GCS) and the National Institute of Health Stroke Scale (NIHSS) were used to determine the stroke severity while the functional outcome was evaluated by the Modified Rankin Score (mRS). Stroke death and stroke recurrence during admission and within 3 months after stroke were also recorded. Poor functional outcome was considered in patients with $\mathrm{mRS}>2$ within the first 3 months after stoke discharge. Follow-up was done daily for clinical evaluation and complications were noted. Oxygen was administrated if ambient oxygen saturation was less than 94\%. Paracetamol was used at $1 \mathrm{~g}$ every six hours if temperatures were above $37.5^{\circ} \mathrm{C}$. Prevention of deep venous thrombosis and stress ulcers was done using a prophylactic dose of enoxaparin (40 mg) and omeprazole $(20 \mathrm{mg})$, respectively. An insulin protocol was set up when capillary glycemia was above $1.4 \mathrm{~g} / \mathrm{L}$. Concerning the blood pressure, nicardipine was given intravenously with an electric syringe in case of high blood pressure with a target of 140 to $160 \mathrm{mmHg}$ for systolic blood pressure in hemorrhagic stroke. In ischemic stroke, early elevated blood pressure was respected except when this was more than $220 \mathrm{mmHg}$ for systolic pressure. Aspirin (100-250 mg per day) was given in ischemic stroke while the curative dose of low weight molecular heparin $(0.1 \mathrm{~mL} / \mathrm{kg}$ twice daily) was used in case of atrial fibrillation with CHADS VASC score $>2$, presence of intraluminal thrombus in a cerebral artery, or presence of blood clot in the heart. Antibiotics and artemether were used for bacterial infection and malaria, respectively. Thrombolysis is not yet effective in Cameroon.

2.3. Statistical Analysis. Statistical analysis was done using the Statistical Package for the Social Sciences (SPSS) standard version, Release 20.0 (IBM Inc., 2012). Mean and standard deviation (SD) of all continuous data are reported. SUA was a scale variable but was categorized into two groups, those having normal and high SUA levels (hyperuricemia) as follows: normal SUA range was SUA $\leq 60 \mathrm{mg} / \mathrm{l}$ in females and SUA $\leq 70 \mathrm{mg} / \mathrm{l}$ in males while high SUA range was SUA > $60 \mathrm{mg} / \mathrm{l}$ in females and SUA > $70 \mathrm{mg} / \mathrm{l}$ in males. SUA was also divided into quintiles (mean SUA level per quintile range) as follows: $Q 1 \leq 49 \mathrm{mg} / \mathrm{l}(41.92 \mathrm{mg} / \mathrm{l}), \mathrm{Q} 2$ $=50-60 \mathrm{mg} / \mathrm{l}(55.41 \mathrm{mg} / \mathrm{l}), \mathrm{Q} 3=61-70 \mathrm{mg} / \mathrm{l}(65.83 \mathrm{mg} / \mathrm{l}), \mathrm{Q} 4$ $=71-84 \mathrm{mg} / \mathrm{l}(76.91 \mathrm{mg} / \mathrm{l})$, and $\mathrm{Q} 5 \geq 85 \mathrm{mg} / \mathrm{l}(105.45 \mathrm{mg} / \mathrm{l})$. Independent samples $t$-test was used to assess differences in continuous variables since the normality assumption was not violated following Kolmogorov-Smirnov and ShapiroWilk test for normality. Cramer's $V$, chi-square test, and Fisher's exact test were used for categorical variables. Univariate analysis was first performed with demographic characteristics and the cerebrovascular risk factors by crosstabulations with $X^{2}$ or Fisher's exact tests for the unadjusted odds ratios (ORs) and then multiple logistic regression was done to adjust the confounding effects of the dependent predictors of death. All predictor variables with $p$ values $<0.2$ that were purposefully gotten from the univariate analysis were included in our multivariate analysis. Survival analysis was performed using Kaplan-Meier and Cox regression analysis. Level of significance was considered 0.05 (two-sided). 
TABLE 1: Basic characteristics and cerebrovascular risk factors (CVRFs) of stroke.

\begin{tabular}{|c|c|}
\hline Variables & Values \\
\hline Age, years $($ mean $\pm S D)$ & $60.58 \pm 13.27$ \\
\hline Males, $n(\%)$ & $389(55.5)$ \\
\hline Ischemic stroke, $n(\%)$ & $480(68.5)$ \\
\hline Cardioembolic stroke, $n(\%)$ & $108(22.5)$ \\
\hline Atherosclerosis stroke, $n(\%)$ & $292(60.8)$ \\
\hline Lacunar stroke, $n(\%)$ & $55(11.5)$ \\
\hline Undetermined, $n(\%)$ & $25(5.2)$ \\
\hline Hemorrhagic stroke, $n(\%)$ & $221(31.5)$ \\
\hline Hypertension, $n(\%)$ & $664(94.7)$ \\
\hline Diabetes mellitus, $n(\%)$ & $240(34.2)$ \\
\hline Smoking, $n(\%)$ & $591(84.3)$ \\
\hline Alcohol abuse, $n(\%)$ & $257(36.7)$ \\
\hline Dyslipidemia, $n(\%)$ & $526(75.0)$ \\
\hline Obesity, $n(\%)$ & $203(29.0)$ \\
\hline Metabolic syndrome, $n(\%)$ & $169(24.1)$ \\
\hline Coronary artery disease, $n(\%)$ & $41(5.8)$ \\
\hline Atrial fibrillation, $n(\%)$ & $93(13.3)$ \\
\hline Heart failure (cardiomyopathy), $n(\%)$ & $91(13.0)$ \\
\hline Valvulopathy, $n(\%)$ & $39(5.6)$ \\
\hline Past stroke, $n(\%)$ & $151(21.5)$ \\
\hline Sedentary lifestyle, $n(\%)$ & $130(18.5)$ \\
\hline Family history of stroke, $n(\%)$ & $22(3.1)$ \\
\hline Hyperuricemia, $n(\%)$ & $327(46.6)$ \\
\hline Gout, $n(\%)$ & $22(3.1)$ \\
\hline Chronic kidney disease, $n(\%)$ & $43(6.1)$ \\
\hline HIV seropositive, $n(\%)$ & $25(3.6)$ \\
\hline
\end{tabular}

\section{Results}

3.1. Baseline Characteristics of Patients. We recruited a total of 701 acute stroke patients among whom 389 (55.5\%) were male patients giving a $\mathrm{M}: \mathrm{F}$ ratio of $1.25: 1(5: 4)$. The age range was from 23 to 100 years with a mean age of $60.58 \pm 13.27$ years. Of the 701 participants, $480(68.5 \%)$ had ischemia. $18(2.6 \%)$ out of 480 ischemic stroke patients presented with a transient ischemic attack (TIA) on admission. HTN was the most predominant CVRF in 664 (94.7\%). 523 (74.6\%) out of the 664 patients with HTN had a known history of HTN while $141(20.1 \%)$ were not known to be hypertensive patients but had acutely elevated blood pressure $>130 / 85 \mathrm{mmHg}$ and even required antihypertensive agents during admission. HTN was insignificantly more predominant among ischemic stroke patients $(450,93.8 \%)$ as compared to hemorrhagic stroke patients $(214,96.8 \%)(p=0.09)$. The proportion of stroke patients with a history of DM, dyslipidemia, obesity, smoking, alcohol consumption, CAD, atrial fibrillation, cardiopathy $(\mathrm{CHF})$, valvular heart disease, previous stroke, and sedentary lifestyle are also shown in Table 1 . The prevalence of hyperuricemia among acute stroke patients was 46.6\% (327/701). The clinical and laboratory data of patients with ischemic and hemorrhagic stroke were compared (Table 2).

3.2. Outcome in the Acute Phase and 90 Days after Stroke. The outcome of patients within the 90 days after stroke has been assessed in Figure 1. In this series, 133 out of 701 patients (19.0\%) died during admission and 568 patients (81.0\%) discharged home alive were eligible for follow-up among whom $10.6 \%$ (60/568) died within three months and $26.1 \%$ (148/568) were lost to follow-up. Among stroke survivors ( $n=360$ ), 62.8\% and $37.2 \%$ had good and bad functional outcomes. Overall, the 3-month poststroke mortality was $34.9 \%$ (193/701). The mean SUA concentration \pm SD (standard deviation) of stroke survivors $(65.73 \pm 21.96 \mathrm{mg} / \mathrm{l})$ was significantly lower than that of those who died (75.07 \pm $28.41 \mathrm{mg} / \mathrm{l}, p$ value $<0.001)$. The mean $\pm \mathrm{SD}$ duration of hospital stay and of follow-up was $8.8 \pm 6.2$ days and $53.6 \pm$ 40.0 days, respectively. On univariate analysis, there was a significant association between high SUA levels and death among acute stroke patients $(p<0.001, \mathrm{OR}=2.067,95 \% \mathrm{CI}$ : 1.449-2.950).

3.3. Predictive Value of SUA on Mortality among Acute Stroke Patients (Table 3). On univariate analysis, the following were factors significantly associated with death in acute stroke patients: history of DM, smoking, alcohol abuse, dyslipidemia, atrial fibrillation, cardiomyopathy (CHF), CKD, hemorrhagic stroke type, hyperglycemia, low HDLc, increased triglycerides, high TG, hyperuricemia, GCS less than 9, NIHSS $>14, \mathrm{mRS}>2$, and presence of in-hospital complications $(p<0.05)$. In the significant association 
TABLE 2: Baseline clinical profile of acute stroke patients.

\begin{tabular}{|c|c|c|c|}
\hline Variables & Ischemic stroke & Hemorrhagic stroke & $p$ value \\
\hline Age, mean \pm SD & $62.77 \pm 13.32$ & $55.81 \pm 11.86$ & $<0.001$ \\
\hline Systolic blood pressure, mean \pm SD & $159.77 \pm 30.532$ & $178.92 \pm 34.437$ & $<0.001$ \\
\hline Diastolic blood pressure, mean \pm SD & $95.32 \pm 19.389$ & $107.62 \pm 22.138$ & $<0.001$ \\
\hline Pulse, mean \pm SD & $83.837 \pm 18.7469$ & $86.223 \pm 20.3571$ & 0.128 \\
\hline Temperature, mean \pm SD & $37.329 \pm 0.8933$ & $37.772 \pm 3.5019$ & 0.010 \\
\hline $\mathrm{O}_{2}$ saturation, mean $\pm \mathrm{SD}$ & $95.68 \pm 4.764$ & $94.69 \pm 5.175$ & 0.027 \\
\hline Height, mean \pm SD & $4.1672 \pm 14.8528$ & $4.0214 \pm 13.8822$ & 0.959 \\
\hline Weight, mean \pm SD & $81.8439 \pm 19.052$ & $86.8788 \pm 18.989$ & 0.181 \\
\hline Body mass index, mean \pm SD & $31.8915 \pm 6.7657$ & $30.3858 \pm 5.6557$ & 0.572 \\
\hline Glasgow Coma Score, mean \pm SD & $13.42 \pm 2.531$ & $11.46 \pm 3.866$ & $<0.001$ \\
\hline NIHSS Score, mean \pm SD & $10.42 \pm 7.837$ & $16.51 \pm 10.177$ & $<0.001$ \\
\hline Modified Rankin Score, mean \pm SD & $2.796 \pm 1.5182$ & $3.710 \pm 1.3972$ & $<0.001$ \\
\hline Serum uric acid, mean \pm SD & $71.058 \pm 25.3186$ & $63.341 \pm 22.1664$ & $<0.001$ \\
\hline White blood cell count, mean \pm SD & $7.3827 \pm 4.68110$ & $8.5558 \pm 4.01643$ & 0.001 \\
\hline Creatinine, mean \pm SD & $16.1565 \pm 26.125$ & $18.0011 \pm 43.412$ & 0.487 \\
\hline Urea, mean \pm SD & $0.5795 \pm 1.162$ & $0.6688 \pm 1.825$ & 0.435 \\
\hline $\mathrm{CRP}$, mean $\pm \mathrm{SD}$ & $63.155 \pm 72.766$ & $74.624 \pm 81.649$ & 0.213 \\
\hline $\mathrm{ESR}$, mean $\pm \mathrm{SD}$ & $45.30 \pm 40.800$ & $53.61 \pm 40.736$ & 0.193 \\
\hline Glycemia, mean \pm SD & $1.4891 \pm 0.89088$ & $1.4185 \pm 0.74641$ & 0.307 \\
\hline Glycated hemoglobin, mean \pm SD & $9.025 \pm 10.285$ & $6.802 \pm 1.798$ & 0.243 \\
\hline Total cholesterol, mean \pm SD & $1.9600 \pm 0.02665$ & $1.9700 \pm 0.2608$ & 0.245 \\
\hline Triglycerides, mean \pm SD & $1.4054 \pm 0.21183$ & $1.1185 \pm 0.04916$ & 0.390 \\
\hline HDL cholesterol, mean \pm SD & $0.4946 \pm 0.25125$ & $0.5187 \pm 0.21349$ & 0.245 \\
\hline LDL cholesterol, mean \pm SD & $1.2860 \pm 0.51114$ & $1.2941 \pm 0.62535$ & 0.864 \\
\hline Hematocrit, mean \pm SD & $38.478 \pm 7.1100$ & $40.460 \pm 6.7768$ & 0.001 \\
\hline Length of hospital stay (days) & $8.400 \pm 6.000$ & $9.720 \pm 9.260$ & 0.053 \\
\hline Duration of follow-up (days) & $55.330 \pm 39.550$ & $49.900 \pm 40.861$ & 0.100 \\
\hline
\end{tabular}

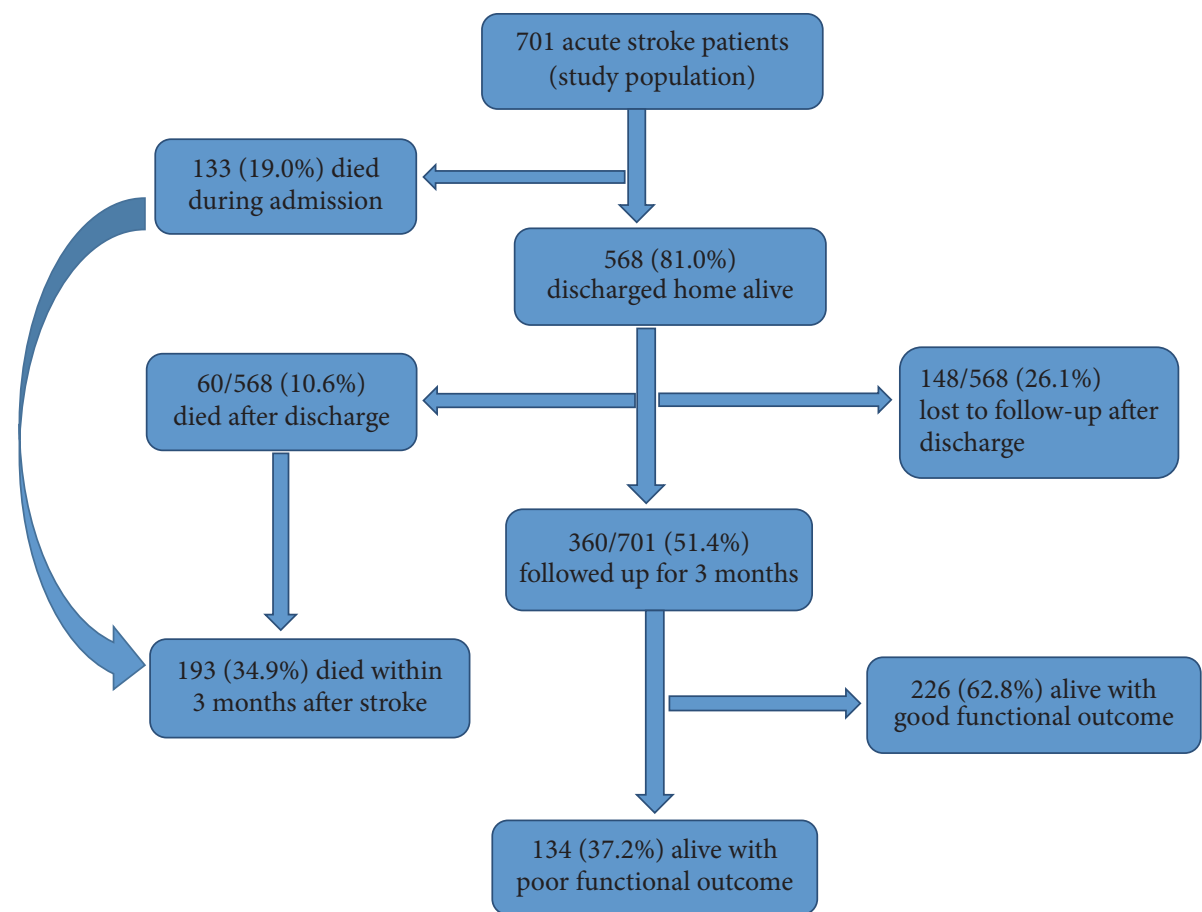

FIGURE 1: Flow chart of functional outcome and death during the 3-month follow-up. 
TABLE 3: Independent predictors of death within 3 months after stroke.

\begin{tabular}{|c|c|c|c|c|c|c|}
\hline Predictors & Alive & Died & Unadjusted OR (95\% CI) & $p$ value & Adjusted OR (95\% CI) & $p$ value \\
\hline Age $>45$ years & $306(63.9)$ & $173(18.9)$ & $1.527(0.84-2.635)$ & 0.127 & $0.984(0.451-2.147)$ & 0.967 \\
\hline Male gender & $206(66.7)$ & $103(33.3)$ & $0.856(0.602-1.216)$ & 0.384 & - & - \\
\hline HTN & $343(65.1)$ & $184(34.9)$ & $1.013(0.443-2.318)$ & 0.975 & - & - \\
\hline $\mathrm{DM}$ & $111(57.2)$ & $83(42.8)$ & $1.693(1.178-2.432)$ & 0.004 & $0.984(0.506-1.913)$ & 0.961 \\
\hline Smoking & $70(76.7)$ & $21(23.1)$ & $0.506(0.300-0.853)$ & 0.010 & $0.839(0.372-1.895)$ & 0.673 \\
\hline Alcohol & $151(70.9)$ & $62(29.1)$ & $0.665(0.454-0.946)$ & 0.024 & $0.768(0.417-1.412)$ & 0.395 \\
\hline Dyslipidemia & $285(68.8)$ & $129(31.2)$ & $0.530(0.358-0.786)$ & 0.001 & $0.602(0.305-1.187)$ & 0.143 \\
\hline Obesity & $113(71.1)$ & $46(28.9)$ & $0.684(0.459-1.019)$ & 0.061 & $0.429(0.103-1.778)$ & 0.243 \\
\hline METS & $93(69.9)$ & $40(30.1)$ & $0.751(0.492-1.143)$ & 0.180 & $1.705(0.359-8.103)$ & 0.502 \\
\hline CAD/IHD & $23(67.6)$ & $11(32.4)$ & $0885(0.422-1.858)$ & 0.748 & - & - \\
\hline AFIB & $34(49.3)$ & $35(50.7)$ & $2.124(1.275-3.533)$ & 0.003 & $3.198(1.386-7.376)$ & 0.006 \\
\hline Cardiopathy & $36(52.9)$ & $32(47.1)$ & $1.789(1.072-2.986)$ & 0.025 & $1.129(0.483-2.642)$ & 0.779 \\
\hline Valvulopathy & $20(66.7)$ & $10(33.3)$ & $0.929(0.426-2.027)$ & 0.853 & - & - \\
\hline Previous stroke & $71(62.3)$ & $43(37.7)$ & $1.167(0.761-1.788)$ & 0.479 & - & - \\
\hline CKD & $17(43.7)$ & $22(56.4)$ & $2.596(1.343-5.017)$ & 0.003 & $2.139(0.829-5.522)$ & 0.116 \\
\hline Hemorrhagic stroke & $104(53.1)$ & $92(46.9)$ & $2.242(1.560-3.223)$ & $<0.001$ & $1.211(0.635-2.307)$ & 0.561 \\
\hline $\mathrm{SBP}>140 \mathrm{mmHg}$ & $276(63.6)$ & $158(36.4)$ & $1.574(0.885-2.134)$ & 0.156 & $1.751(0.803-3.821)$ & 0.159 \\
\hline $\mathrm{DBP}>90 \mathrm{mmHg}$ & $227(62.4)$ & $137(37.6)$ & $1.867(1.225-2.854)$ & 0.061 & $0.801(0.405-1.586)$ & 0.525 \\
\hline Hyperglycemia & $107(52.2)$ & $98(47.8)$ & $2.439(1.699-3.502)$ & $<0.001$ & $2.263(1.203-4.256)$ & 0.011 \\
\hline High LDLc & $270(66.0)$ & $139(34.0)$ & $0.858(0.578-1.273)$ & 0.447 & - & - \\
\hline Low HDLc & $210(70.9)$ & $86(24.7)$ & $0.574(0.403-0.817)$ & 0.002 & $0.673(0.394-1.148)$ & 0.146 \\
\hline High TC & $170(62.7)$ & $101(37.3)$ & $1.227(0.865-1.741)$ & 0.252 & - & - \\
\hline High TG & $70(51.1)$ & $67(48.9)$ & $2.203(1.484-3.270)$ & $<0.001$ & $1.857(1.003-3.437)$ & 0.049 \\
\hline Hyperuricemia & $148(56.5)$ & $114(40.7)$ & $2.067(1.449-2.950)$ & $<0.001$ & $1.265(0.707-2.263)$ & 0.428 \\
\hline $\mathrm{GCS} \leq 8$ & $8(8.7)$ & $84(91.3)$ & $33.908(15.916-72.242)$ & $<0.001$ & $7.604(3.221-17.948)$ & $<0.001$ \\
\hline NIHSS $>14$ & $60(27.6)$ & $157(72.4)$ & $21.806(13.820-34.407)$ & $<0.001$ & $6.733(3.659-12.390)$ & $<0.001$ \\
\hline $\mathrm{mRS}>2$ & $184(50.0)$ & $184(50.0)$ & $19.556(9.708-39.392)$ & $<0.001$ & $3.363(1.456-7.767)$ & 0.005 \\
\hline Complications & $108(46.6)$ & $124(53.4)$ & $4.193(2.894-6.075)$ & $<0.001$ & $1.631(0.943-2.821)$ & 0.080 \\
\hline
\end{tabular}

METS: metabolic syndrome; AFIB: atrial fibrillation; CKD: chronic kidney disease; CAD/IHD: coronary artery/ischemic heart disease.

observed between hyperuricemia and death among acute stroke patients on univariate analysis, patients with elevated SUA after stroke had unadjusted odds ratio $(95 \% \mathrm{CI})$ of $2.067(1.449-2.950)(p<0.001)$ of mortality compared with normouricemic patients. However, on multivariate analysis, no such independent association was seen $(\mathrm{OR}=1.265$ (CI: 0.707-2.263); $p=0.428$ ). After multivariate analysis, only atrial fibrillation, hyperglycemia, hypertriglyceridemia, GCS less than 9, mRS greater than 2, and NIHSS greater than 14 remained as significant independent predictors of death within 3 months after stroke in acute stroke patients $(p<0.05)$. However, an independent association between increasing SUA concentration and survival was noted with the Kaplan-Meier curve with adjusted HR (95\% CI) of $1.740(1.305-2.320), p<0.001$, as shown in Figure 2. The proportion of death within 3 months after stroke significantly increased with high levels of SUA $(p<0.001)$ ranging from $30(15.5 \%)$ deaths in the lowest SUA quintile range $(Q 1)$ to $56(29.0 \%)$ deaths in the 4 th quintile range (Q4) and 48 deaths $(24.5 \%)$ in the highest SUA quintile range (Q5). The curves in Figure 3 show that death within 3 months after stroke in acute stroke significantly increases as SUA levels increase among stroke patients. This is shown in Figure 3.

3.4. Predictive Value of SUA on Functional Outcome among Acute Stroke Patients (Table 4). On univariate analysis, there was a significant association between high SUA levels and poor outcome among acute stroke patients $(p<0.001, \mathrm{OR}$ $=3.268$, 95\% CI: 2.093-5.104). On univariate analysis, the following were also significant predictors of poor functional outcomes in all acute stroke patients: older patients $>45$ years, unemployment, recurrent stroke, hyperuricemia, low GCS $(\leq 8)$, NIHSS $>14, \mathrm{mRS}>2$, elevated serum urea, and presence of in-hospital complications $(p<0.05)$. On multivariate analysis, there was a significant association between hyperuricemia and poor functional outcomes among acute stroke patients, where patients with elevated SUA after stroke had adjusted odds ratio of 2.482 (95\% CI: 1.399-4.404; $p=$ 0.002 ) of mortality compared with normouricemic patients (Table 4). 


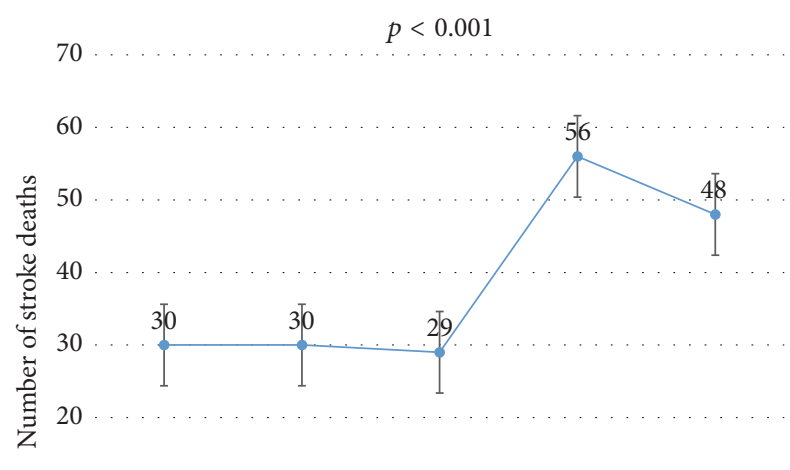

10

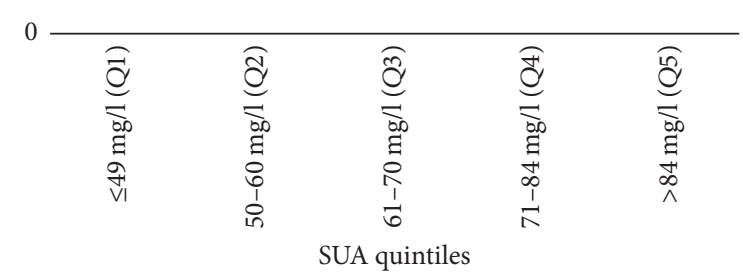

FIGURE 2: Death rate across SUA quintiles.

\section{Discussion}

In our study, the prevalence of hyperuricemia among acute stroke patients was $46.6 \%$ (327/701); we observed that even though high SUA was not an independent predictor of stroke death, death rate significantly increased across the higher SUA quintile ranges in acute stroke patients. The findings from this observational prospective study support the notion that SUA is an adverse prognostic marker of stroke outcome among black African patients.

In Ghana, Sarfo et al. reported a high frequency of hyperuricemia of $46.3 \%$ among Ghanaian stroke patients which was similar to that seen in our study [17]. Similarly, Masoud et al., in Iran [18], reported the mean SUA levels in 55 patients (46 ischemic and 9 hemorrhagic stroke patients) studied to be $59.4 \pm 17.0 \mathrm{mg} / \mathrm{l}$ and about half of the patients (47.3\%) were hyperuricemic. In India, Kotwal et al. reported a slightly lower prevalence of hyperuricemia among 100 acute stroke patients in 2015 [19]. This very slight discrepancy could due to the differences in methodology such as timing of SUA measurement. The prevalence of hyperuricemia in the United States' general population is $20.1 \%$ according to a large 10-year follow-up study reported by the National Health and Nutrition Examination Survey (NHANES), 2007-2008 [20]. Another study in Bangkok's population showed that prevalence of hyperuricemia is $24.4 \%$ [21] and a study in a developing country reported the prevalence of hyperuricemia to be $35.2 \%$ in men and $8.7 \%$ in women [22]. In India, Koppula et al. showed that $24.7 \%$ of controls in their study were hyperuricemic [23]. According to these studies, the prevalence of hyperuricemia is significantly higher in patients with acute stroke than in the normal population.

Regarding the association between stroke mortality and hyperuricemia in our study, we noted that hyperuricemia was associated with stroke mortality and was a predictor

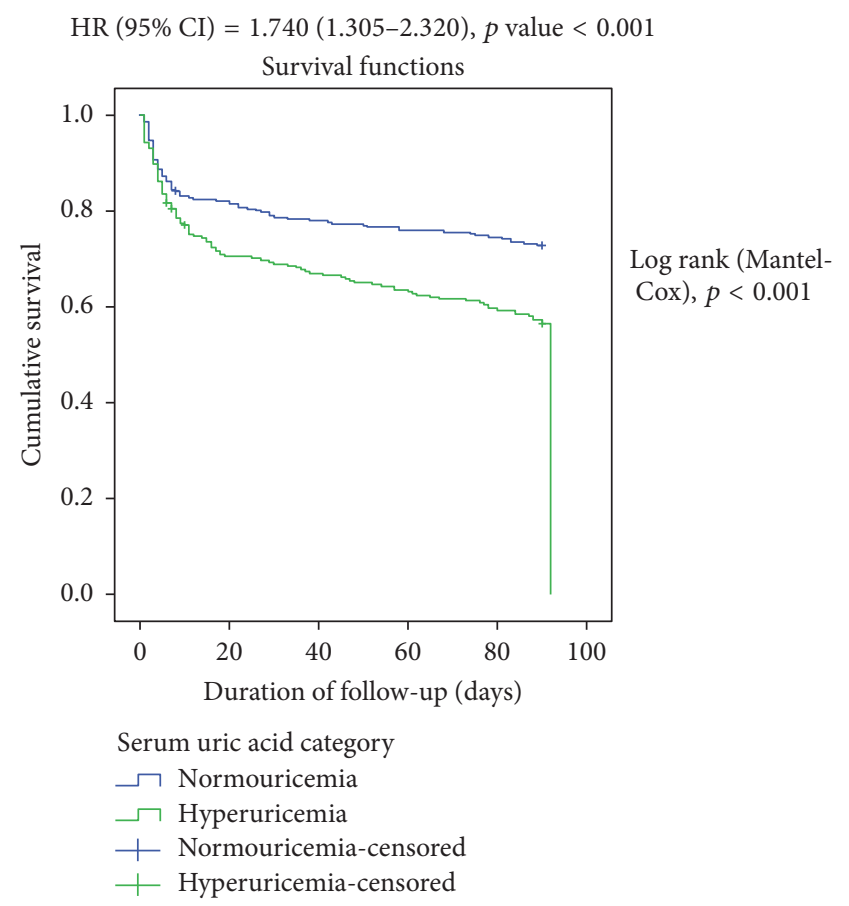

FIGURE 3: A graph showing a Kaplan-Meier survival rate of patients with normouricemia compared with hyperuricemia after an acute stroke.

of poor functional outcome among acute stroke patients. Similarly, in the recent Ghanaian prospective observational cohort, SUA concentration was positively correlated with stroke severity and an association was observed between stroke mortality and hyperuricemia [17]. In a longitudinal study including 418 patients in Congo, reported by LongoMbenza et al., hyperuricemia was found to be a predictor of stroke and all-cause mortality [24]. Karagiannis et al. found that elevated SUA was strongly associated with early death among 435 patients presenting with stroke in Greece [25]. Furthermore, stroke death rate significantly increased across the higher SUA quintile ranges in both ischemic stroke and all stroke patients. Similarly, Strasak et al. in a longterm prospective study demonstrated that the highest SUA quintile concentration was significantly related to mortality from $\mathrm{CHF}$ and stroke [26]. Our findings are to a greater extent also consistent with the body of previous scientific evidence which supports the fact that SUA is an adverse predictor of early stroke death and functional outcome [5, 27-30]. In experimental and in vivo conditions, it has been shown that SUA can act as a proinflammatory molecule [31]. A direct mechanistic role for hyperuricemia in atherogenesis and the clinical course of cerebrovascular disease has been suggested by the links between elevated SUA and increased production of oxygen free radicals [32], accelerated oxygenation of low-density lipoproteins which facilitates lipid peroxidation $[33,34]$, impairment of nitric oxide production with subsequent activation of the renin-angiotensin system [35], and induction of endothelial dysfunction and smooth muscle cell proliferation [36, 37]. Furthermore, uric acid increases platelet adhesiveness and aggregation $[32,38]$, stimulates the 
TABLE 4: Independent predictors of poor functional outcome within 3 months post stroke.

\begin{tabular}{|c|c|c|c|c|c|c|}
\hline Predictors & Good outcome & Poor outcome & Unadjusted OR (95\% CI) & $p$ value & Adjusted OR (95\% CI) & $p$ value \\
\hline Age $>45$ years & $185(60.5)$ & $13(24.1)$ & $2.063(1.061-4.010)$ & 0.030 & $2.306(0.966-5.508)$ & 0.060 \\
\hline Male gender & $132(64.1)$ & $74(35.4)$ & $0.878(0.571-1.352)$ & 0.555 & - & - \\
\hline Unemployed & $123(68.0)$ & $58(32.0)$ & $0.639(0.416-0.983)$ & 0.041 & $1.075(0.593-1.948)$ & 0.811 \\
\hline Unmarried (single) & $62(68.1)$ & 29 (31.9) & $0.731(0.441-1.210)$ & 0.222 & - & - \\
\hline No health insurance & $67(67.7)$ & $32(32.3)$ & $0.745(0.457-1.214)$ & 0.236 & - & - \\
\hline HTN & $212(61.8)$ & $131(38.2)$ & $2.884(0.813-10.226)$ & 0.087 & $2.860(0.670-12.211)$ & 0.156 \\
\hline $\mathrm{DM}$ & $66(59.5)$ & $45(40.5)$ & $1.226(0.775-1.940)$ & 0.385 & - & - \\
\hline Smoking & $40(57.1)$ & $30(42.9)$ & $1.341(0.789-2.281)$ & 0.277 & - & - \\
\hline Alcohol & $95(62.9)$ & $56(37.1)$ & $0.990(0.642-1.527)$ & 0.964 & - & - \\
\hline Dyslipidemia & $175(61.4)$ & $110(38.6)$ & $1.336(0.778-2.294)$ & 0.293 & - & - \\
\hline Obesity & $69(61.1)$ & $44(38.9)$ & $1.112(0.703-1.759)$ & 0.649 & - & - \\
\hline METS & $56(60.2)$ & $37(39.8)$ & $1.158(0.713-1.880)$ & 0.553 & - & - \\
\hline CAD/IHD & $16(69.6)$ & $7(30.4)$ & $0.723(0.290-1.806)$ & 0.486 & - & - \\
\hline AFIB & $20(58.8)$ & $14(41.2)$ & $1.202(0.585-2.467)$ & 0.616 & - & - \\
\hline Cardiopathy & $25(69.4)$ & $11(30.6)$ & $0.719(0.342-1.512)$ & 0.383 & - & - \\
\hline Valvulopathy & $14(70.0)$ & $6(30.0)$ & $0.710(0.266-1.894)$ & 0.492 & - & - \\
\hline Recurrent stroke & $32(45.1)$ & $39(54.9)$ & $2.488(1.468-4.220)$ & 0.001 & $2.566(1.247-5.280)$ & 0.010 \\
\hline CKD & $11(64.7)$ & $6(35.3)$ & $0.916(0.331-2.537)$ & 0.866 & - & - \\
\hline Hemorrhagic stroke & $63(60.6)$ & $41(39.4)$ & $1.141(0.714-1,822)$ & 0.582 & - & - \\
\hline $\mathrm{SBP}>140 \mathrm{mmHg}$ & $174(63.0)$ & $102(37.0)$ & $0.953(0.576-1.576)$ & 0.850 & - & - \\
\hline $\mathrm{DBP}>90 \mathrm{mmHg}$ & $142(62.6)$ & $85(37.4)$ & $1.026(0.659-1.598)$ & 0.909 & - & - \\
\hline Hyperglycemia & $59(55.1)$ & $48(44.9)$ & $1.580(0.996-2.506)$ & 0.051 & $1.253(0.670-0.670)$ & 0.480 \\
\hline High LDLc & $165(61.1)$ & $105(38.9)$ & $1.339(0.808-2.219)$ & 0.257 & - & - \\
\hline Low HDLc & $129(61.4)$ & $81(38.6)$ & $1.149(0.744-1.776)$ & 0.531 & - & - \\
\hline High TC & $106(62.4)$ & $64(37.6)$ & $1.035(0.675-1.588)$ & 0.875 & - & - \\
\hline High TG & $45(64.3)$ & $25(35.7)$ & $0.823(0.536-1.589)$ & 0.771 & - & - \\
\hline Hyperuricemia & $69(46.6)$ & $79(53.4)$ & $3.268(2.093-5.104)$ & $<0.001$ & $2.482(1.399-4.404)$ & 0.002 \\
\hline GCS $<9$ & $1(12.5)$ & $7(87.5)$ & $12.402(1.509-101.943)$ & 0.005 & $4.306(0.439-42.263)$ & 0.210 \\
\hline NIHSS $>14$ & $18(30.0)$ & $42(70.0)$ & $5.275(2.883-9.653)$ & $<0.001$ & $1.369(0.657-2.854)$ & 0.402 \\
\hline $\mathrm{mRS}>2$ & $67(36.4)$ & $117(63.6)$ & $16.333(9.114-29.269)$ & $<0.001$ & $14.639(7.591-28.232)$ & $<0.001$ \\
\hline Complications & $53(49.1)$ & $55(50.9)$ & $2.273(1.432-3.606)$ & $<0.001$ & $1.033(0.552-1.936)$ & 0.919 \\
\hline High urea & $40(50.0)$ & $40(50.0)$ & $1.968(1,189-3.257)$ & 0.008 & $1.037(0.532-2.020)$ & 0.916 \\
\hline High creatinine & $56(58.9)$ & 39 (41.1) & $1.239(0.767-2.002)$ & 0.381 & - & - \\
\hline
\end{tabular}

METS: metabolic syndrome; AFIB: atrial fibrillation; CKD: chronic kidney disease; CAD/IHD: coronary artery/ischemic heart disease.

synthesis of monocyte chemoattractant protein-1 (MCP-1) in rat vascular smooth muscles via mitogen-activated protein kinase and cyclooxygenase-2 [39], and stimulates mice mononuclear cells to produce IL-1 $\beta$, IL-6, and tumor necrosis factor- $\alpha$ (TNF- $\alpha)$ [40]. All these factors are important for the development and progression of atherosclerosis and may therefore explain the strong association between uric acid and carotid atherosclerosis, a well-known risk factor for stroke $[8,41]$

Despite the overwhelming evidence from this current and previous epidemiological studies, some studies still demonstrate contradictory findings. In a study carried out by Amaro et al., higher SUA levels were associated with an increased rate of excellent recovery independently of baseline variables [10]. Still in Spain, Chamorro et al. found out that diabetic patients had lower SUA values and there was a $12 \%$ increase in the odds of good clinical outcome for each milligram per deciliter increase of SUA in patients with acute ischemic stroke [42]. Wu et al. (2013) conducted a study on 1351 ischemic and 380 cerebral hemorrhage patients, which showed that decreased uric acid levels correlated with poor outcomes in acute ischemic stroke patients, but not in cerebral hemorrhage patients [43]. According to a study conducted in 585 young Chinese patients with acute ischemic stroke by Zhang et al., in 2010, it was observed that elevated SUA is an independent predictor for good clinical outcome of acute cerebral infarction among young adults and, unlike our study, lower SUA levels at time of admission were observed more frequently in the lowest quintile for patients with severe stroke [44]. Therefore, these findings support the fact that high SUA concentration could be neuroprotective and has antioxidant properties in the setting of an acute stroke.

Despite these intriguing differences, the findings of this study support the fact that SUA is a poor prognostic marker 
or predictor of mortality and functional outcome among acute stroke Cameroonian patients. SUA reduction is a viable and promising strategy to reduce stroke mortality and poor functional outcome.

\section{Conclusion}

The prevalence of hyperuricemia in stroke is quite high as about 1 in 2 acute stroke patients had hyperuricemia on admission. Hyperuricemia is associated with stroke mortality and is a predictor of adverse functional outcomes in acute stroke. These results therefore suggest that prospective studies should be conducted to determine whether reducing SUA levels after acute ischemic stroke would be beneficial within our setting.

\section{Conflicts of Interest}

The authors declare that they have no conflicts of interest.

\section{References}

[1] G. A. Donnan, M. Fisher, M. Macleod, and S. M. Davis, "Stroke," The Lancet, vol. 371, no. 9624, pp. 1612-1623, 2008.

[2] V. L. Feigin, M. H. Forouzanfar, and R. Krishnamurthi, "Global and regional burden of stroke during 1990-2010: findings from the Global Burden of Disease Study 2010," The Lancet, vol. 383, no. 9913, pp. 245-254, 2014.

[3] J. H. Chin, "Stroke in sub-saharan africa: an urgent call for prevention," Neurology, vol. 78, no. 13, pp. 1007-1008, 2012.

[4] R. Beaglehole, R. Bonita, G. Alleyne et al., "UN high-level meeting on non-communicable diseases: addressing four questions," The Lancet, vol. 378, no. 9789, pp. 449-455, 2011.

[5] C. J. Weir, S. W. Muir, M. R. Walters, and K. R. Lees, "Serum urate as an independent predictor of poor outcome and future vascular events after acute stroke," Stroke, vol. 34, no. 8, pp. 19511956, 2003.

[6] A. Cherubini, C. Ruggiero, M. C. Polidori, and P. Mecocci, "Potential markers of oxidative stress in stroke," Free Radical Biology and Medicine, vol. 39, no. 7, pp. 841-852, 2005.

[7] B. F. Becker, "Towards the physiological function of uric acid," Free Radical Biology and Medicine, vol. 14, no. 6, pp. 615-631, 1993.

[8] F. J. Nieto, C. Iribarren, M. D. Gross, G. W. Comstock, and R. G. Cutler, "Uric acid and serum antioxidant capacity: a reaction to atherosclerosis?" Atherosclerosis, vol. 148, no. 1, pp. 131-139, 2000.

[9] Y. Wang, H. Wu, Q. Jia et al., "Decreased uric acid levels correlate with poor outcomes in acute ischemic stroke patients, but not in cerebral hemorrhage patients," Journal of Stroke and Cerebrovascular Diseases, vol. 23, no. 3, pp. 469-475, 2014.

[10] S. Amaro, X. Urra, M. Gómez-Choco et al., "Uric acid levels are relevant in patients with stroke treated with thrombolysis," Stroke, vol. 42, no. 1, pp. S28-S32, 2011.

[11] W. Yq, J. L, X. Yx, W. Yl, L. Yy, H. Dy et al., "Predictive value of serum uric acid on cardiovascular disease and all-cause mortality in urban Chinese patients," Chinese Medical Journal, vol. 123, no. 11, pp. 1387-1391, Jun 2010.

[12] A. M. Strasak, C. C. Kelleher, L. J. Brant et al., "Serum uric acid is an independent predictor for all major forms of cardiovascular death in 28,613 elderly women: A prospective 21-year follow-up study, International Journal of Cardiology, vol. 125, no. 2, pp. 232-239, 2008.

[13] M. Madero, M. J. Sarnak, X. Wang et al., "Uric acid and long-term outcomes in CKD," The American Journal of Kidney Diseases, vol. 53, no. 5, pp. 796-803, 2009.

[14] I. Holme, A. H. Aastveit, N. Hammar, I. Jungner, and G. Walldius, "Uric acid and risk of myocardial infarction, stroke and congestive heart failure in 417734 men and women in the Apolipoprotein MOrtality RISk study (AMORIS)," Journal of Internal Medicine, vol. 266, no. 6, pp. 558-570, 2009.

[15] N. Y. Mapoure, C. B. Tchaleu Nguenkam, H. B. Mbatchou Ngahane et al., "Predictors of in-hospital mortality for stroke in Douala, Cameroon," Stroke Research and Treatment, Article ID 681209, 2014.

[16] W. L. Williams, Third Report of the National Cholesterol Education Program (NCEP) Expert Panel on Detection, Evaluation, and Treatment of High Blood Cholesterol in Adults (Adult Treatment Panel III) Final Report, Circulation, 2002.

[17] F. Sarfo, J. Akassi, N. Antwi et al., "Highly prevalent hyperuricaemia is associated with adverse clinical outcomes among Ghanaian stroke patients: An observational prospective study," Ghana Medical Journal, vol. 49, no. 3, p. 165, 2015.

[18] M. Masoud, K. Mahbubeh, N. Neda, R. Mohamad, and F. SeyedMohammad, "Serum uric acid level in acute stroke patients," Medical Journal of Islamic Republic of Iran, vol. 2, pp. 66-72, 2012.

[19] S. K. Kotwal, J. B. Singh, S. Mahajan, and A. Mahajan, "Serum Uric Acid Levels in Patients with Acute Stroke," JK Science, Journal of Medical Education and Research, vol. 17, no. 4, 2017.

[20] Y. Zhu, B. J. Pandya, and H. K. Choi, "Prevalence of gout and hyperuricemia in the US general population: the national health and nutrition examination survey 2007-2008," Arthritis and Rheumatism, vol. 62, 10, pp. 3136-3141, 2011.

[21] S. Uaratanawong, S. Suraamornkul, S. Angkeaw, and R. Uaratanawong, "Prevalence of hyperuricemia in Bangkok population," Clinical Rheumatology, vol. 30, no. 7, pp. 887-893, 2011.

[22] D. Conen, V. Wietlisbach, P. Bovet et al., "Prevalence of hyperuricemia and relation of serum uric acid with cardiovascular risk factors in a developing country," BMC public health, vol. 4, p. 9, 2004.

[23] R. Koppula, S. Kaul, A. Venkateswar Rao, A. Jyothy, and A. Munshi, "Association of serum uric acid level with ischemic stroke, stroke subtypes and clinical outcome," Neurol Asia, vol. 18, no. 4, 2017.

[24] B. Longo-Mbenza, E. Lukoki Luila, P. Mbete, and E. Kintoki Vita, "Is hyperuricemia a risk factor of stroke and coronary heart disease among Africans?" International Journal of Cardiology, vol. 71, no. 1, pp. 17-22, 1999.

[25] A. Karagiannis, D. P. Mikhailidis, K. Tziomalos et al., "Serum uric acid as an independent predictor of early death after acute stroke," Circulation Journal, vol. 71, no. 7, pp. 1120-1127, 2007.

[26] A. Strasak, E. Ruttmann, L. Brant et al., "Serum uric acid and risk of cardiovascular mortality: A prospective long-term study of 83683 Austrian men," Clinical Chemistry, vol. 54, no. 2, pp. 273-284, 2008.

[27] M. S. Babu, S. Kaul, S. Dadheech, K. Rajeshwar, A. Jyothy, and A. Munshi, "Serum albumin levels in ischemic stroke and its subtypes: Correlation with clinical outcome," Nutrition, vol. 29, no. 6, pp. 872-875, 2013. 
[28] A. Papazafiropoulou, A. Sotiropoulos, E. Skliros, M. Kardara, A. Kokolaki, and S. Pappas, "Predictors of In-hospital mortality after acute ischemic stroke in subjects with and without diabetes mellitus," The Open General \& Internal Medicine Journal, vol. 3, no. 1, pp. 34-39, 2009.

[29] E. Chiquete, J. L. Ruiz-Sandoval, L. M. Murillo-Bonilla et al., "Serum uric acid and outcome after acute ischemic stroke: premier study," Cerebrovascular Diseases, vol. 35, no. 2, pp. 168174, 2013.

[30] J. Dawson, Prevention of stroke: risk stratification and targeted and novel therapies, University of Glasgow, 2009, http://theses .gla.ac.uk/id/eprint/851.

[31] S. Amaro, A. M. Planas, and Á. Chamorro, "Uric acid administration in patients with acute stroke: A novel approach to neuroprotection," Expert Review of Neurotherapeutics, vol. 8, no. 2, pp. 259-270, 2008.

[32] R. J. Johnson, D.-H. Kang, D. Feig et al., "Is there a pathogenetic role for uric acid in hypertension and cardiovascular and renal disease?" Hypertension, vol. 41, no. 6, pp. 1183-1190, 2003.

[33] V. Schlotte, A. Sevanian, P. Hochstein, and K. U. Weithmann, "Effect of uric acid and chemical analogues on oxidation of human low density lipoprotein in vitro," Free Radical Biology and Medicine, vol. 25, no. 7, pp. 839-847, 1998.

[34] B. Karten, U. Beisiegel, G. Gercken, and A. Kontush, "Mechanisms of lipid peroxidation in human blood plasma: A kinetic approach," Chemistry and Physics of Lipids, vol. 88, no. 2, pp. 83-96, 1997.

[35] P. Eslami, D. B. Corry, M. D. Nyby, and M. L. Tuck, "Inhibition of oxidative stress and improvement of nitric oxide production by ACE inhibitors and AT1 receptor blockers in uric acid stimulated vascular smooth muscle cells," American Journal of Hypertension, 5, pp. S154-S155, 2004.

[36] U. M. Khosla, S. Zharikov, J. L. Finch et al., "Hyperuricemia induces endothelial dysfunction," Kidney International, vol. 67, no. 5, pp. 1739-1742, 2005.

[37] G. N. Rao, M. A. Corson, and B. C. Berk, "Uric acid stimulates vascular smooth muscle cell proliferation by increasing plateletderived growth factor A-chain expression," The Journal of Biological Chemistry, vol. 266, no. 13, pp. 8604-8608, 1991.

[38] M. H. Ginsberg, F. Kozin, M. O’Malley, and D. J. McCarty, "Release of platelet constituents by monosodium urate crystals," Journal of Clinical Investigation, vol. 60, no. 5, pp. 999-1007, 1977.

[39] J. Kanellis, S. Watanabe, J. H. Li et al., "Uric acid stimulates monocyte chemoattractant protein-1 production in vascular smooth muscle cells via mitogen-activated protein kinase and cyclooxygenase-2," Hypertension, vol. 41, no. 6, pp. 1287-1293, 2003.

[40] M. G. Netea, B. J. Kullberg, W. L. Blok, R. T. Netea, and J. W. M. Van der Meer, "The role of hyperuricemia in the increased cytokine production after lipopolysaccharide challenge in neutropenic mice," Blood, vol. 89, no. 2, pp. 577-582, 1997.

[41] J. R. Crouse, J. F. Toole, W. M. McKinney et al., "Risk factors for extracranial carotid artery atherosclerosis," Stroke, vol. 18, no. 6, pp. 990-996, 1987.

[42] A. Chamorro, V. Obach, A. Cervera, M. Revilla, R. Deulofeu, and J. H. Aponte, "Prognostic significance of uric acid serum concentration in patients with acute ischemic stroke," Stroke, vol. 33, no. 4, pp. 1048-1052, 2002.

[43] H. Wu, Q. Jia, G. Liu, L. Liu, Y. Pu, and X. Zhao, Stroke Cerebrovascular Disc, 2013 Jun.
[44] B. Zhang, C. Gao, N. Yang et al., "Is elevated SUA associated with a worse outcome in young Chinese patients with acute cerebral ischemic stroke?" BMC Neurology, vol. 10, article no. 82, 2010. 


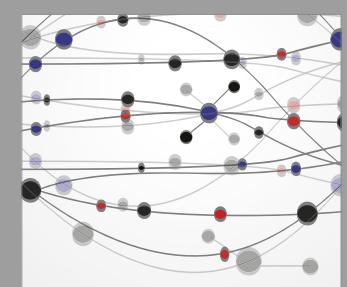

The Scientific World Journal
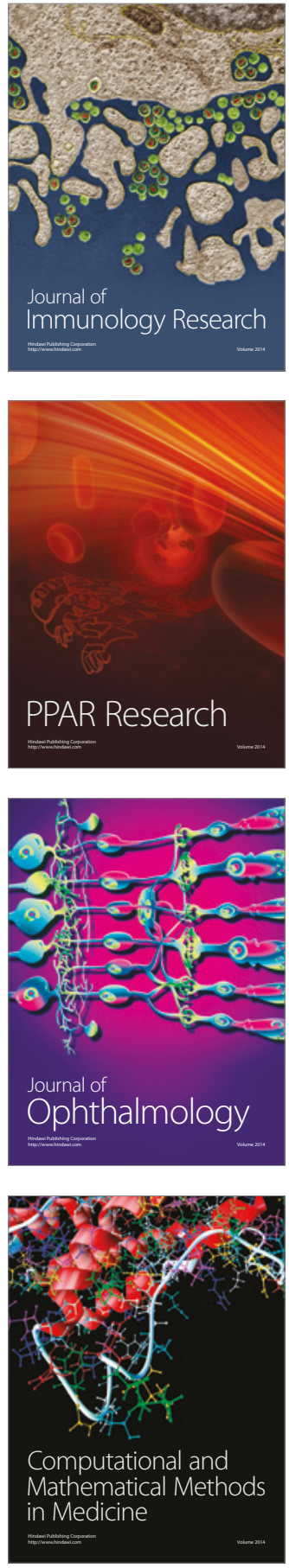

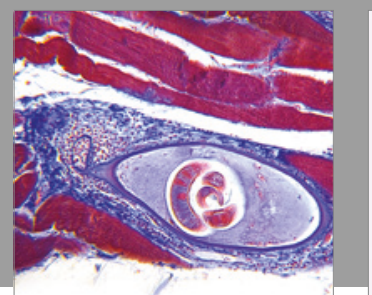

Gastroenterology Research and Practice
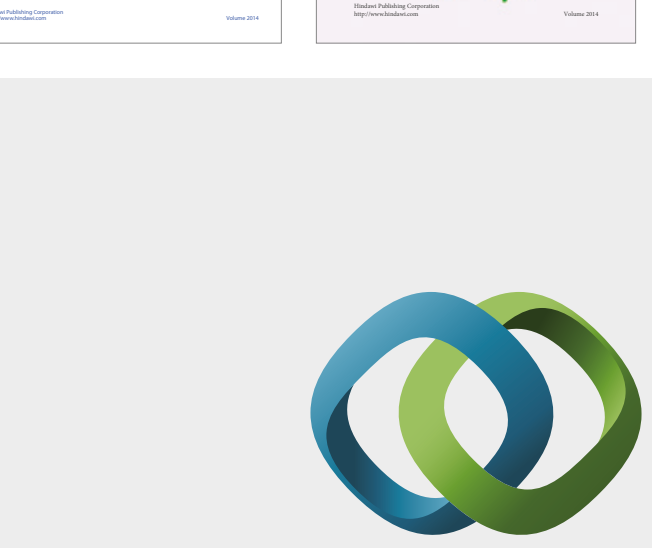

\section{Hindawi}

Submit your manuscripts at

https://www.hindawi.com
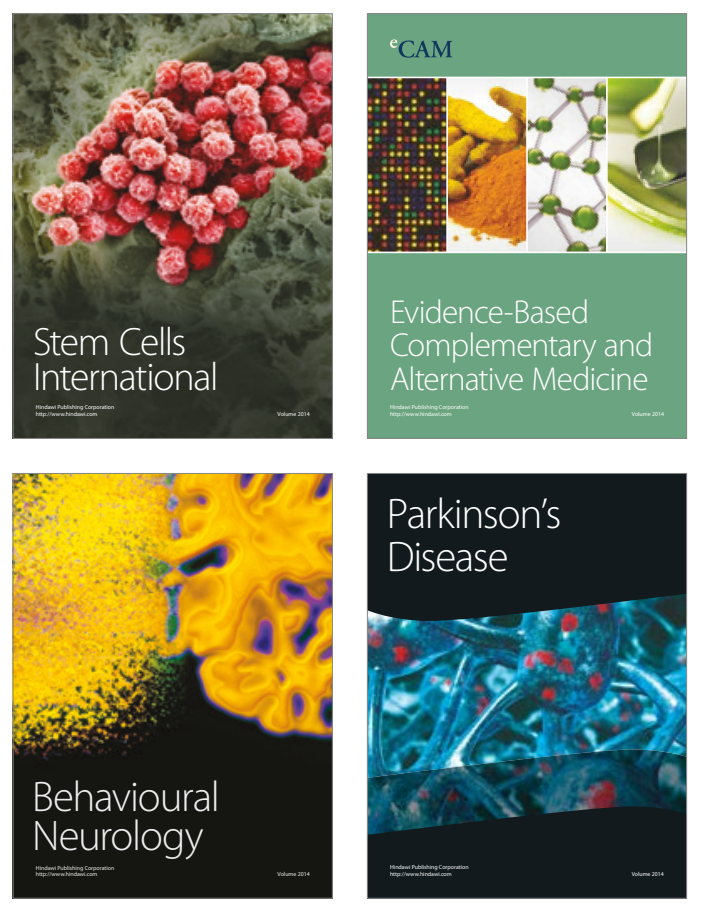
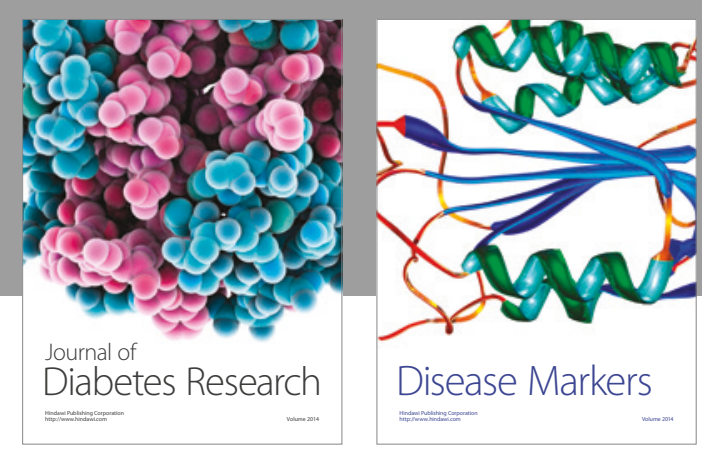

Disease Markers
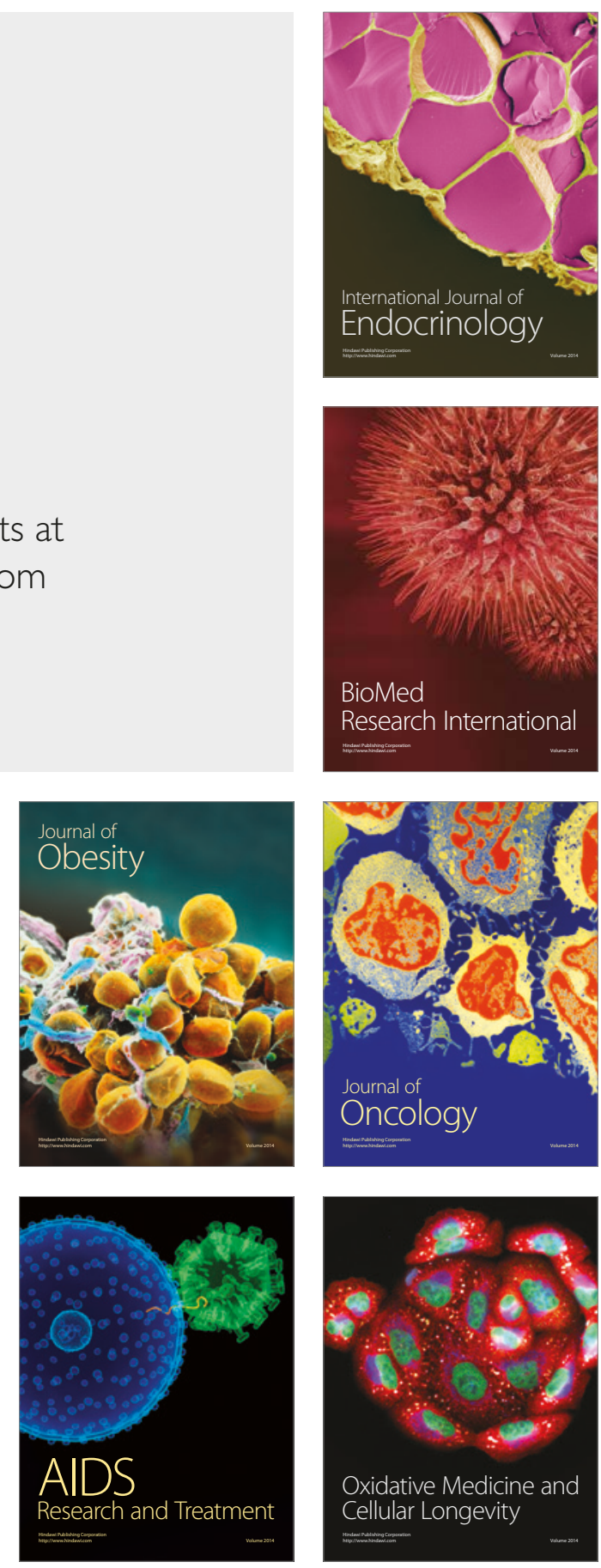